González-Fimbres, R.A.; Ramírez-Siqueiros, M.G.; Vaca-Rubio, H.; Moueth-Cabrera, M.T. y Hernández-Cruz, G. (2020) Relationship Between Post-Exercise hrv and Internal Training Load in Triathletes. Revista Internacional de Medicina y Ciencias de la Actividad Física y el Deporte vol. 20

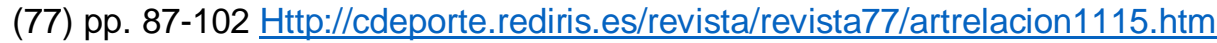

DOI: $10.15366 /$ rimcafd2020.77.006

\title{
ORIGINAL
}

\section{RELACIÓN ENTRE VFC POST-EJERCICIO Y LA CARGA INTERNA DE ENTRENAMIENTO EN TRIATLETAS}

\section{RELATIONSHIP BETWEEN POST-EXERCISE HRV AND INTERNAL TRAINING LOAD IN TRIATHLETES}

González-Fimbres, R.A. ${ }^{\text {; }}$ Ramírez-Siqueiros, M.G. ${ }^{1}$; Vaca-Rubio, H. ${ }^{2}$; MouethCabrera, M.T. ${ }^{1}$ y Hernández-Cruz, G. ${ }^{3}$

1 Profesores Investigadores de Tiempo Completo, Licenciatura en Entrenamiento Deportivo, Universidad Estatal de Sonora (México) roberto.gonzalez@ues.mx, grethel.ramirez@ues.mx, terecesues@gmail.com

2 Profesor de asignatura, Licenciatura en Entrenamiento Deportivo, Universidad Estatal de Sonora (México) humberto.vaca@hotmail.com

3 Profesor Investigador de Tiempo Completo, Facultad de Organización Deportiva, Universidad Autónoma de Nuevo León (México) german.hernandezcrz@uanl.edu.mx

\section{AGRADECIMIENTOS / ACKNOWLEDGEMENTS}

Queremos agradecer a los entrenadores del equipo de triatlón de la Comisión del Deporte del Estado de Sonora por permitirnos llevar a cabo este estudio.

We want to thank Mexico's Sonora State Sports Commission's triathlon team coaches for allowing us to carry out this work.

Código UNESCO / UNESCO Code: 2411 Fisiología Humana / Human Physiology, 241106 Fisiología del ejercicio / Exercise Physiology.

Clasificación del consejo de Europa / Europe's Council Classification: 6. Fisiología del ejercicio / Exercise Physiology.

Recibido 2 de abril de 2018 Received April 2, 2018

Aceptado 1 de octubre de 2018 Accepted October 1, 2018

\section{RESUMEN}

El objetivo del estudio fue relacionar valores de VFC post-ejercicio con componentes de carga interna de entrenamiento medida por tres métodos de Estímulo de 
Entrenamiento (Training Impulse, TRIMP) en triatletas juveniles. Los métodos de TRIMP estuvieron relacionados entre ellos ( $r=0,970$ a $0,983, R^{2}=0.940$ a 0.967$)$, sin embargo, mostraron diferencias significativas en sus valores promedio ( $p<$ $0.01)$. El entrenamiento redujo significativamente la VFC $(p<0.01)$ y existe un efecto significativo del tiempo en la recuperación de la VFC $(p<0.01)$. La reducción de la VFC en Post5 está negativamente relacionada con la intensidad del ejercicio, volumen en $>90 \%$ FCres y valores de TRIMP $(r=-0.302$ a $-0.495, p<0.01)$ y positivamente relacionada con el volumen en $<50 \%$ FCres $(r=0.488, p<0.01)$. La reducción post-ejercicio de la VFC puede proporcionar información valiosa para evaluar efectos fisiológicos del entrenamiento interválico o continuo en triatletas juveniles.

PALABRAS CLAVE: Sistema nervioso autónomo, TRIMP, ejercicio, respuestas autónomas, recuperación autónoma.

\section{ABSTRACT}

The aim of the study was to stablish relationships between post-exercise heart rate variability (HRV) values and internal training load (TL) components measured by three Training Impulse (TRIMP) methods in youth triathletes. TRIMP methods were related with each other $\left(r=0,970\right.$ to $0,983, \mathrm{R}^{2}=0,940$ to 0,967$)$ but showed significant differences in mean values $(p<0,01)$. Training significantly reduced HRV $(p<0,01)$ with a significant time effect on HRV recovery $(p<0,01)$. Post5 $\mathrm{HRV}$ reduction has a negative relationship to exercise intensity, volume at $>90 \%$ HRres, and TRIMP values $(r=-0,302$ to $-0,495, p<0,01)$ and a positive relationship with volume at $<50 \%$ HRres $(r=0.488, p<0,01)$. Immediate postexercise HRV reduction can provide valuable information for evaluation of physiological effects at intervallic or continuous training in youth triathletes.

KEYWORDS: Autonomic Nervous System, TRIMP, exercise, autonomic responses, autonomic recovery.

\section{INTRODUCCIÓN}

La cuantificación de la carga interna es una parte importante del proceso de entrenamiento. El entrenador debe verificar que el estímulo físico es lo suficientemente grande para activar mecanismos de compensación, así como que no exceda la capacidad de recuperación del atleta ${ }^{1}$. Los métodos de evaluación de carga interna basados en frecuencia cardiaca (FC), conocidos como estímulo de entrenamiento (Training impulse, TRIMP), se utilizan frecuentemente debido a su naturaleza objetiva y practicidad en escenarios de campo. Banister introdujo este concepto en $1991^{2}$ calculando en TRIMP con la duración del ejercicio en minutos multiplicado por el promedio de la FC de reserva (FCres) obtenida durante esa sesión de entrenamiento y ponderada por la intensidad del ejercicio utilizando la relación exponencial entre la elevación fraccional de la FC y la concentración de 
lactato sanguíneo (LS) ${ }^{3,4}$. Aunque se ha reportado que este método es útil para la actividad continua, se dice que no puede discriminar entre sesiones de entrenamiento continuas e intermitentes que tengan la misma duración y $\mathrm{FC}$ promedio, y que no es válido para entrenamiento interválico de alta intensidad con alta contribución anaeróbica ${ }^{5}$.

Para atender este problema se han referenciado varios métodos en la literatura. El TRIMP de Edwards utiliza cinco zonas de entrenamiento arbitrarias basadas en el porcentaje de FC máxima (FCmax), multiplicando el tiempo transcurrido en cada zona por factores de ponderación arbitrarios ${ }^{6}$. El TRIMP de Lucia utiliza tres zonas de entrenamiento basadas en los umbrales ventilatorios con factores de ponderación arbitrarios ${ }^{7}$. Existen referencias a otro método similar al de Lucia, pero en lugar de utilizar umbrales ventilatorios, los autores evaluaron umbrales de lactato 8,9. El TRIMP de Stagno definió cinco zonas de entrenamiento por medio de parámetros fisiológicos y asignó factores de ponderación de acuerdo a la ecuación de regresión de la curva de lactato utilizando datos promedio del equipo ${ }^{10}$. EI TRIMP de Manzi, considerado como el más avanzado, asigna factores de ponderación individualizados a cada valor de FC observado durante el ejercicio ${ }^{11}$.

Akubat, Patel y Abt revisaron estos métodos ${ }^{12}$ y notaron algunas limitaciones: a) El uso de zonas de entrenamiento puede ya sea subestimar o sobreestimar la carga interna cuando los valores de FC de ejercicio se acercan a los límites de las zonas; b) Las zonas de entrenamiento del TRIMP de Edwards no tienen fundamento fisiológico; c) Los TRIMP de Lucia y Edwards no proveen un fundamento fisiológico para establecer los factores de ponderación; d) El factor de ponderación del TRIMP de Stagno no refleja respuestas individuales. El único método que aparenta superar estas limitaciones es el TRIMP de Manzi. Sin embargo, para que entrenadores y atletas puedan utilizar este método ellos deben de considerar el tiempo requerido, altos costos, acceso instalaciones de laboratorio, equipamiento y personal calificado.

La variabilidad de la FC (VFC) es un marcador fisiológico de evaluación del sistema nervioso autónomo (SNA), tanto para la rama simpática como la parasimpática y se usa comúnmente en estudios clínicos y de investigación debido a su aparente facilidad de cálculo ${ }^{13}$. Tres métodos pueden ser utilizados para el análisis de la VFC: dominios de tiempo, dominios de frecuencia y no lineales ${ }^{13}$. Se piensa que los métodos de dominio de frecuencia no son confiables en contextos de atletas muy entrenado ${ }^{5}$, ya que estos índices son afectados por la frecuencia respiratoria (FR) y pueden llevar a una falsa clasificación de fatiga ${ }^{14}$. Por lo tanto, la mayoría de los estudios recientes prefieren utilizar métodos de dominio de tiempo, en particular la raíz cuadrada de la media de la suma de los cuadrados de las diferencias entre los intervalos $R-R$ adyacentes (RMSSD) ya que representa la variabilidad a corto plazo de la VFC y la modulación parasimpática, parece no ser afectada por la FR y por su confiabilidad y sensibilidad de detectar adaptaciones fisiológicas ${ }^{5}$. Un estudio reciente propone un nuevo índice de VFC, el Stress Score (SS), el cual se piensa que representa la actividad simpática ${ }^{15}$. Anterior a ese 
estudio, no encontramos ningún índice que se propusiera como un indicador de solo actividad simpática. El SS se basa en un método no lineal, el gráfico de Poincaré. El eje transversal del gráfico (SD1) refleja los cambios a corto plazo de los intervalos $\mathrm{R}-\mathrm{R}$ y se cree que tiene una relación directamente proporcional a la actividad parasimpática ${ }^{15}$. El significado del eje longitudinal (SD2) no está definido claramente, pero parece ser inversamente proporcional a la actividad parasimpática 15. Se puede calcular el SS con la inversa del SD2 y establecer una relación simpática/parasimpática ${ }^{15}$.

En escenarios deportivos, generalmente se usa la VFC en reposo para monitorear el estatus de SNA. Se cree que las fluctuaciones de VFC están relacionadas con la adaptación a la carga de entrenamiento ${ }^{16}$. Aunque la mayoría de los estudios utilizan valores matutinos en reposo, ha crecido el interés en monitorear los efectos agudos que tiene la carga de entrenamiento en la VFC post-ejercicio. Se dice que el entrenamiento físico cambia el SNA a un impulso simpático y a un retiro parasimpático ${ }^{17,18}$. Y aunque los valores de VFC durante el ejercicio tienden a cero sin importar el volumen o la intensidad ${ }^{19}$, varios estudios concuerdan en que la reducción inmediata de la VFC post-ejercicio (de los primeros 2 a 5 minutos de recuperación) se ve afectada principalmente por la intensidad del ejercicio que por el volumen o la duración ${ }^{5,20,21}$.

También se ha estudiado el ritmo de la recuperación de la VFC post-ejercicio, debido a que puede ser explicada por una reducción en la actividad simpática con un aumento simultáneo de la activación del nervio vago ${ }^{5}$. Varios estudios afirman que el factor principal que afecta el retraso de la recuperación de la VFC es la intensidad del ejercicio ${ }^{20,22-24}$ y que este no se ve afectado por el volumen de entrenamiento ${ }^{22,23}$. Cuando los sujetos entrenan a intensidades bajas (por debajo del primer umbral ventilatorio) se puede observar una rápida recuperación de los indicies de VFC post-ejercicio ${ }^{24}$. En entrenamientos de alta intensidad (por encima del segundo umbral ventilatorio) se observa un retraso en la recuperación de los índices de VFC, lo cual se puede atribuir a la naturaleza anaeróbica de la actividad, probablemente explicada por respuestas metabolo-reflejas ${ }^{20,24}$. La noción de que es la intensidad y no el volumen de entrenamiento lo que más afecta el retraso de la recuperación de la VFC puede ser engañoso, ya que los estudios revisados comparan tratamientos con diferentes duraciones en el mismo rango de intensidad baja. Sería interesante ver si la recuperación de la VFC se ve afectada al cambiar la duración de ejercicios de alta intensidad. Por otro lado, varios estudios sostienen que la recuperación de la VFC post-ejercicio también se ve afectada por el estatus de entrenamiento ${ }^{21,22}$. Además, se ha declarado que la modalidad del ejercicio (intermitente vs continuo) afecta la recuperación de la VFC, pero estos resultados son ambiguos, dado que las comparaciones fueron hechas entre tratamientos ejecutados a la misma intensidad de ejercicio ${ }^{25}$.

Aunque la mayoría de los estudios que han medido la VFC post-ejercicio inmediata han considerado el volumen y la intensidad, encontramos que la literatura que compare estos valores con valores de TRIMP es incipiente. La mayoría de los 
estudios comparan al TRIMP con VFC en reposo para evaluar las respuestas de actividad autónoma y la adaptación al ejercicio ${ }^{18,26}$. Sin embargo, un estudio estableció una relación negativa entre los TRIMP de Manzi y Banister con valores de VFC post-ejercicio en los tiempo 60, 120 y 180 segundos ${ }^{23}$. En otro trabajo se propuso un novedoso método de TRIMP basado en VFC para ejercicio continuo, observando la reducción de la VFC y la recuperación a los 30 minutos como una función de la intensidad de entrenamiento, multiplicada por la duración en minutos 5. Este método mostró correlación significativa y acuerdo con el TRIMP de Banister utilizando tratamientos en condiciones controladas de intensidad y duración. Por lo tanto, el objetivo de este estudio fue analizar la relación entre la estimación de carga interna con diferentes métodos de TRIMP y varios índices de VFC post-ejercicio en triatletas juveniles que participan en un régimen real de entrenamiento.

\section{MATERIALES Y MÉTODOS}

\section{SUJETOS}

Siete triatletas juveniles de nivel nacional, cuatro hombres (edad $15 \pm 0,8$ años, estatura $167,6 \pm 2,6 \mathrm{~cm}$, masa corporal $54,9 \pm 1,9 \mathrm{Kg}$; \% grasa $12,7 \pm 1,7)$ y tres mujeres (edad $15 \pm 2,7$ años; estatura 165,3 $\pm 6,7 \mathrm{~cm}$; masa corporal $51,5 \pm 3,9 \mathrm{Kg}$; $\%$ grasa $20,5 \pm 2,5$ ), fueron invitados a participar en el estudio. Los datos fueron recolectados durante una fase de preparación de un macrociclo de entrenamiento para el campeonato nacional. Fueron excluidos los sujetos que tuvieran cualquier condición que pudiera alterar los resultados. Ya que todos los sujetos eran menores de edad, tanto ellos como sus tutores legales firmaron voluntariamente un consentimiento informado expresando su acuerdo en participar. El estudio fue aprobado por el Comité de Bioética en Investigación en Ciencias de la Salud del Centro de Investigación y Desarrollo en Ciencias de la Salud de la Universidad Autónoma de Nuevo León con Folio COBICIS-58/12/2017/02-FOD-BRRC. Todos los procedimientos estuvieron en acuerdo con los lineamientos establecidos por la Declaración del Helsinki.

\section{DISEÑO}

La duración total del estudio fueron dos semanas. Los sujetos realizaron dos sesiones de entrenamiento de carrera al día, uno en la mañana y otro en la tarde. Durante este periodo se abstuvieron de entrenar ciclismo y natación. La FC se monitoreó durante todas las sesiones de entrenamiento. A cada sujeto se le sometió a mediciones de VFC antes y después de cada sesión de entrenamiento en posición sentado. El estudio tuvo carácter observacional.

\section{METODOLOGÍA}

La FCmax fue determinada utilizando una prueba de campo de esfuerzo incremental (test de Leger) en carrera hasta el agotamiento ${ }^{27}$. La FC fue registrada durante la prueba, el valor máximo observado fue considerado como la FCmax. La FC mínima 
(FCmin) se asumió como el valor más bajo observado en un periodo de monitoreo en reposo de 5 minutos antes de la prueba ${ }^{3}$.

Las mediciones de VFC consistieron en el registro de intervalos R-R en posición sentado ${ }^{23}$. Se instruyó a los sujetos para que se abstuvieran de moverse o hablar durante la medición. Los datos fueron recolectados y registrados utilizando un monitor de FC validado capaz de registrar intervalos R-R (Polar Team2, Finlandia). Todas las series $R-R$ registradas por lo monitores de FC fueron extraídas a una computadora personal por medio del programa de procesamiento (Polar Team2, Polar Electro) a una hoja de cálculo Excel Se hizo una identificación visual de latidos ectópicos ocasionales y fueron reemplazados por valores de intervalos $R-R$ adyacentes interpolados. Se utilizó el software Kubios (Universidad de Finlandia Oriental, Kuopio, Finalndia) para calcular todos los parámetros de VFC ${ }^{15}$.

Las mediciones de VFC en cada sesión de entrenamiento se hicieron en dos momentos diferentes, 5 minutos antes del calentamiento (Pre5), y durante 20 minutos posterior a la sesión de entrenamiento. Para un análisis subsecuente, esta segunda medición se dividió en cuatro periodos de 5 minutos (0-5 minutos: Post5, 5-10 minutos: Post10, 10-15 minutos: Post15 y 15-20 minutos: Post20). La medición de VFC a corto plazo de 5 minutos, especialmente la RMSSD, es un parámetro que puede ser utilizado para evaluar la recuperación post-ejercicio como un índice de reactivación parasimpática ${ }^{28}$. Un seguimiento de 5 minutos después del ejercicio parece ser suficiente para diferenciar ejercicios con distinta carga interna ${ }^{23}$.

Para el análisis de los datos de VFC, los dominios de tiempo se restringieron al LnRMSSD ${ }^{5}$. Nos abstuvimos de utilizar marcadores de frecuencia de la VFC por su poca confiablidad en el contexto de atletas altamente entrenados ${ }^{5}$.

En los métodos no lineales obtuvimos los índices de SD1 y SD2 del gráfico de Poincaré. Dos índices adicionales se obtuvieron, el SS y la proporción simpática/parasimpática (S:PS) ${ }^{15}$ calculada de la siguiente manera:

$$
\begin{gathered}
S S=1000 * 1 / S D 2 \\
S: P S=S S / S D 1
\end{gathered}
$$

Tres métodos de TRIMP basados en FC fueron utilizados para estimar la carga de entrenamiento interna. TRIMP de Edwards, TRIMP de Banister y un nuevo TRIMP ajustado propuesto por los autores. El TRIMP de Edwards suma el tiempo en minutos transcurrido en cada una de las cinco zonas discretas de entrenamiento relativas al \%FCmax, un multiplicador se asocia a cada zona de FC dándole una mayor ponderación a los valores más altos de FC ${ }^{6}$. 
El TRIMP de Banister se basa en el promedio de la FCres y la duración del ejercicio 2. El producto de estos factores se pondera por la concentración de LS predicha por una ecuación, la cual es diferente de acuerdo al sexo:

$$
\begin{gathered}
\text { TRIMP de Banister hombres }=T * \frac{F C e j e-F C \min }{F C \max -F C \min } * 0.64 * e^{1.92 * \frac{F C e j e-F C \min }{\text { FCmax }-F C \min }} \\
\text { TRIMP de Banister mujer }=T * \frac{F C e j e-F C \min }{F C \max -F C \min } * 0.86 * e^{1.67 * \frac{F C e j e-F C \min }{F C m a x-F C m i n}}
\end{gathered}
$$

Donde:

$T=$ Duración de la sesión de entrenamiento en minutos.

FCeje = Promedio de la FC de la sesión de entrenamiento en latidos por minuto.

$\mathrm{FCmin}=\mathrm{FC}$ en reposo en latidos por minuto.

FCmax = FC máxima en latidos por minuto.

e = Logaritmo Neperiano de 2,712.

Con propósitos prácticos, se evaluó el desempeño de un nuevo método llamado TRIMP ajustado. Para su cálculo utilizamos la ecuación de Banister ajustada al sexo para ponderar cada valor de FC observado durante el ejercicio a un ritmo de muestreo de 1 segundo, al igual que se hace en el método de Manzi ${ }^{11}$, superando la limitación del TRIMP de Banister de usar la FCres promedio.

Para la validez ecológica de los datos, las sesiones de entrenamiento fueron diseñadas y conducidas por el entrenador de los atletas ${ }^{21}$. Los investigadores nos abstuvimos de participar en el proceso de entrenamiento. Se condujeron dos sesiones diarias de entrenamiento a las 8:00 am y a las 5:00 pm. Las sesiones matutinas consistieron en entrenamiento interválico de alta intensidad y las sesiones vespertinas consistieron en entrenamiento continuo de intensidad baja a moderada.

\section{ANÁLISIS ESTADÍSTICO}

Todos los datos descriptivos se muestran como media \pm DE. Se verificó la normalidad de los datos con la prueba Shapiro-Wilk. Los datos de VFC post-ejercicio fueron transformados por logaritmo y normalizados a un porcentaje de los valores individuales pre-ejercicio. El transcurso del tiempo de la recuperación de la VFC en función de la modalidad de entrenamiento se analizó utilizando una ANOVA de mediciones repetidas de 2 modalidades de entrenamiento por 4 tiempos de recuperación. Para comparar los valores de VFC entre los turnos matutino y vespertino se utilizó una prueba $t$ de muestras relacionadas. Se utilizó el coeficiente de correlación de Pearson para estudiar las relaciones entre los valores de VFC post-ejercicio y el volumen, intensidad y valores de TRIMP. La significancia estadística fue establecida a $p<0,05$. Se utilizó el software SPSS versión 22 (SPSS Inc, Chicago, IL) para realizar todos los análisis estadísticos. 


\section{RESULTADOS}

Los métodos de TRIMP estuvieron altamente correlacionados entre ellos (Figura 1). Se encontraron diferencias significativas entre los tres métodos sin importar si la sesión de entrenamiento fue interválica o continua (Tabla 1).

a)

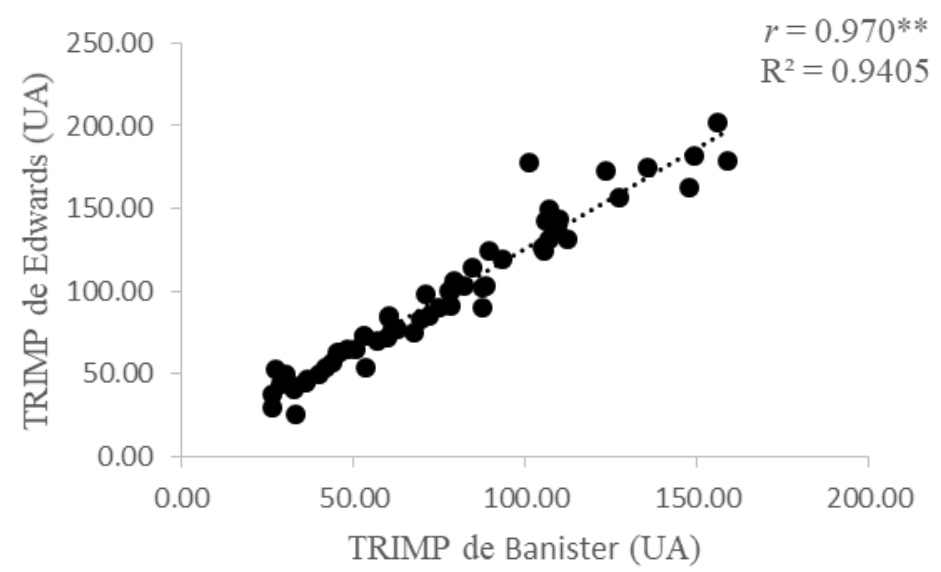

b)

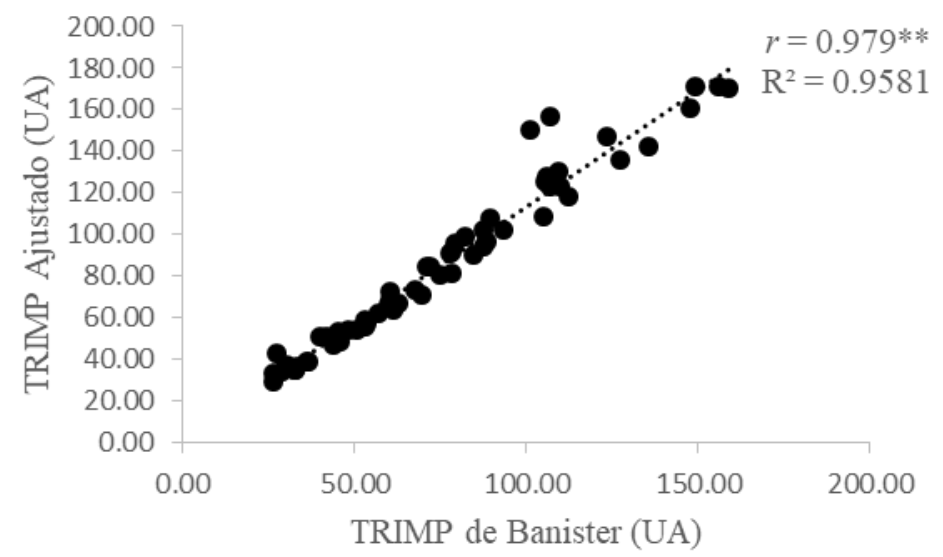

c)

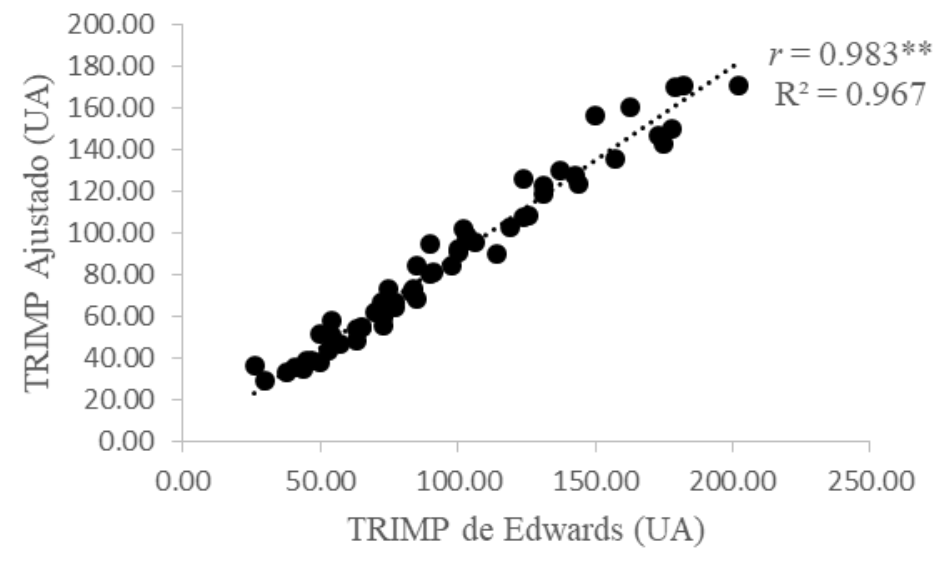

Figura 1. Correlaciones entre los métodos de TRIMP. a) Edwards y Banister, b) Banister y Adjustado, c) Edwards y Adjustado $\mathrm{UA}=$ Unidades arbitrarias; ${ }^{* *}=p<0.001$. 
Rev.int.med.cienc.act.fís.deporte - vol. 20 - número 77 - ISSN: 1577-0354

Tabla 1. Diferencia de medias entre los métodos de TRIMP.

\begin{tabular}{lccc}
\hline $\begin{array}{c}\text { Modalidad de } \\
\text { entrenamiento }\end{array}$ & \multicolumn{3}{c}{ Método de TRIMP } \\
\hline \multirow{3}{*}{ General } & Banister & Ajustado & Edwards \\
\cline { 2 - 4 } Continuo & $75.64 \pm 35.65^{\star *}$ & $86.33 \pm 40.87^{* *}$ & $96.16 \pm 44.58^{\star *}$ \\
Intervalo & $75.01 \pm 37.97^{\star *}$ & $83.74 \pm 42.38^{* *}$ & $94.71 \pm 47.28^{\star *}$ \\
\hline \multicolumn{4}{c}{${ }^{* *}=$ Diferencias entre los métodos $p<0.01}$. \\
\end{tabular}

El comportamiento de los índices de VFC en Pre5, Post5, Post10, Post15 y Post20 puede ser observado en la figura 2. Las sesiones de entrenamiento redujeron significativamente la VFC. Se encontraron diferencias en Ln RMSSD, SS y S:PS entre Pre5 y Post5 $(p<0,01)$. El análisis mostró un efecto significativo del tiempo en la recuperación de la VFC en marcadores parasimpáticos y de balance S:PS ya que todas la mediciones repetidas fueron diferentes entre ellas $(p<0,01)$. El Ln SS, el cual se considera un marcador de actividad simpática, no mostró reducciones significativas hasta Post20. 
Rev.int.med.cienc.act.fís.deporte - vol. 20 - número 77 - ISSN: 1577-0354
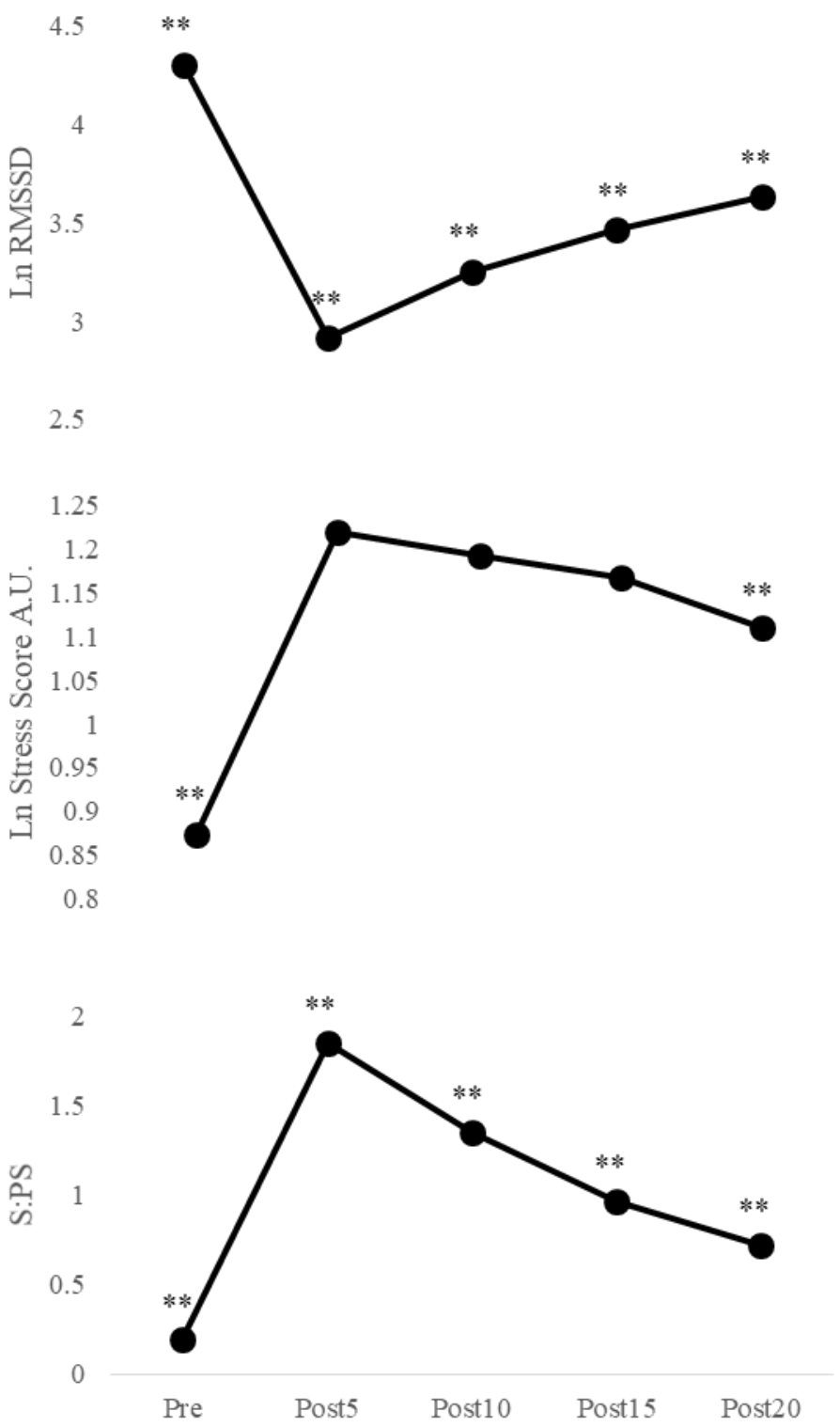

Figura 2. Diferencias entre los valores de VFC en los momentos de muestreo. Ln RMSSD = Logaritmo natural de la RMSSD, Ln Stress Score = Logaritmo natural del Stress Score, S:PS = Proporción Simpática/Parasimpática (Stress Score/SD1); ${ }^{* \star}=p<0.01$.

Los coeficientes de correlación entre la reducción de la VFC relativa en Pre5 Post5, post10, post15 y post20 con la intensidad del ejercicio, volumen y TRIMP se muestran en la tabla 2. La reducción de la Ln RMSSD normalizada en Post5 no tuvo relación con minutos totales, pero mostró relación con volumen en tiempo transcurrido $<50 \%$ FCres y $>92 \%$ FCres, Ninguno de los valores Post5 de SS o $\mathrm{S}: \mathrm{PS}$ tuvieron relación con alguna variable de ejercicio o de carga de entrenamiento interna. 
Tabla 2. Coeficientes de correlación de los índices de VFC y las variables de entrenamiento (Intensidad, volumen y estímulo de entrenamiento).

\begin{tabular}{|c|c|c|c|c|c|c|c|c|}
\hline \multirow[b]{2}{*}{$\begin{array}{c}\text { Valores } \\
\text { normalizados } \\
\text { relativos a Pre5 }\end{array}$} & \multicolumn{2}{|c|}{ Intensidad } & \multicolumn{3}{|c|}{ Volumen } & \multicolumn{3}{|c|}{ TRIMP } \\
\hline & $\begin{array}{c}\text { FC } \\
\text { prom. }\end{array}$ & $\begin{array}{c}\% \\
\text { FCres } \\
\text { prom. }\end{array}$ & $\begin{array}{l}\text { Minutos } \\
\text { totales }\end{array}$ & $\begin{array}{l}<50 \% \\
\text { FCres }\end{array}$ & $\begin{array}{l}>90 \% \\
\text { FCres }\end{array}$ & Banister & Edwards & Ajustado \\
\hline RMSSD Post5 & $-0.47^{\star \star}$ & $-0.49^{* *}$ & 0.03 & $.488^{\star *}$ & $-.030^{\star}$ & $-0.32^{*}$ & $-0.37^{\star \star}$ & $-0.34^{\star \star}$ \\
\hline RMSSD Post10 & $-0.34^{\star *}$ & $-0.35^{\star \star}$ & -0.02 & $.302^{*}$ & $-0.33^{*}$ & -0.19 & -0.26 & -0.23 \\
\hline RMSSD Post15 & -0.21 & -0.23 & -0.02 & .177 & $-0.29^{*}$ & -0.14 & -0.18 & -0.19 \\
\hline RMSSD Post20 & -0.15 & -0.16 & 0.02 & .132 & -0.25 & -0.07 & -0.10 & -0.11 \\
\hline SS Post5 & -0.07 & -0.05 & 0.16 & .079 & 0.25 & 0.01 & 0.07 & 0.09 \\
\hline SS Post 10 & 0.01 & 0.02 & 0.22 & .076 & 0.14 & 0.02 & 0.09 & 0.07 \\
\hline SS Post15 & 0.07 & 0.10 & 0.17 & .020 & 0.25 & 0.08 & 0.13 & 0.14 \\
\hline SS Post20 & 0.11 & 0.14 & 0.20 & -.003 & 0.12 & 0.12 & 0.14 & 0.16 \\
\hline S:PS Post5 & 0.01 & -0.02 & -0.18 & -.066 & 0.05 & -0.11 & -0.09 & -0.09 \\
\hline S:PS Post10 & 0.07 & 0.04 & -0.04 & -.020 & 0.02 & -0.06 & -0.03 & -0.05 \\
\hline S:PS Post15 & 0.06 & 0.04 & -0.11 & -0.03 & 0.04 & -0.07 & -0.07 & -0.05 \\
\hline S:PS Post20 & 0.09 & 0.08 & -0.20 & -0.15 & -0.06 & -0.07 & -0.09 & -0.08 \\
\hline
\end{tabular}

Nota. Los datos de VFC de RMSSD y SS HRV en esta tabla fueron transformados por logaritmo. ${ }^{*}=p<0.05,{ }^{* *}=p<0.01$.

No se encontró relación con ninguna variable de ejercicio o carga de entrenamiento interna y los parámetros de recuperación de la VFC.

\section{DISCUSIÓN}

El objetivo de este estudio fue analizar la relación entre la estimación de carga interna de entrenamiento con diferentes métodos de TRIMP y varios índices de VFC post-ejercicio en triatletas que participan en un régimen real de entrenamiento. También, se hace la propuesta de una nueva metodología de cuantificación de carga de entrenamiento interna ajustando el método de TRIMP de Banister.

Las publicaciones revisadas sostienen que la intensidad del ejercicio es el factor principal para la reducción de la VFC post-ejercicio ${ }^{5,20,25}$ y que ésta no está relacionada con el volumen ${ }^{29}$. Nuestros resultados parecen confirmar esto, dado que la intensidad general, medida como la FC media de la sesión de entrenamiento y como el \%FCres, está significativamente relacionada con la reducción parasimpática inmediata post-ejercicio de la VFC y no se encontró relación entre parámetros de VFC post-ejercicio y volumen considerado como el total de minutos. Sin embargo, pensamos que este acercamiento es incompleto, ya que los estudios revisados sólo comparan tratamientos de diferentes volúmenes en intensidades bajas, y hasta donde sabemos, ninguno de ellos ha comparado diferentes volúmenes a alta intensidad. 
Se dice que la supresión parasimpática cardiaca está mediada por metabolo-reflejo muscular, siendo esto un factor clave en mediciones de índices parasimpáticos de VFC reducidos ${ }^{30}$. También se asume que la duración del ejercicio en intensidades muy bajas (por debajo del primer umbral ventilatorio) aparenta tener poco efecto en la reducción de la actividad parasimpática cardiaca, mientras que el tiempo transcurrido en alta intensidad (por encima del segundo umbral ventilatorio) se asocia con reducidos valores parasimpáticos ${ }^{21}$. Una posible explicación es que debajo del primer umbral ventilatorio se utiliza un sistema energético preponderantemente aeróbico y las concentraciones de LS no rebasan los valores en reposo. Por el contrario, por encima del segundo umbral ventilatorio se utiliza un sistema energético principalmente anaeróbico glucolítico. Esto causa que las concentraciones de LS aumenten exponencialmente disparando metabolo-reflejos que suprimen la actividad parasimpática. Aunque no medimos LS en este estudio, podemos decir que lo anterior concuerda con nuestros resultados, dado que encontramos correlación positiva con índices de VFC post-ejercicio y el tiempo transcurrido en intensidades $<50 \%$ FCres, las cuales es sabido que son predominantemente aeróbicas, y encontramos relación negativa con el tiempo transcurrido $>90 \%$ FCres, que se ha comprobado que incita mayores concentraciones de LS.

Se piensa que el tiempo transcurrido entre el primer y segundo umbral de lactato no parece tener efecto en la reducción de la VFC ${ }^{21}$. Aunque la concentración de LS supera los valores en reposo en estas intensidades, por la combinación del aporte energético entre sistemas aeróbico y anaeróbico el cuerpo es capaz de metabolizar el lactato al mismo ritmo que se produce manteniendo un estado estable. Esto concuerda con nuestros resultados, ya que la reducción de la VFC y el tiempo transcurrido entre el $50 \%$ y el $90 \%$ de la FCres no tuvo relación alguna. Esto contradice el reporte que establece al primer umbral de lactato como un valor binario para las alteraciones cardiacas autónomas ${ }^{22}$.

La recuperación de la VFC se explica por una reducción de la actividad simpática y un aumento de la actividad parasimpática ${ }^{5}$. Aparentemente, todos los estudios que analizan la recuperación post-ejercicio de la VFC solamente han utilizado índices parasimpáticos. Este estudio explora el uso del SS como un índice simpático y la proporción S:PS como un indicador del balance autónomo ${ }^{15}$. Se encontró un efecto significativo del tiempo en la recuperación de Ln RMSSD en Post5, Post10, Post15 y Post20. Se cree que el retiro simpático tiene la mayor contribución para la recuperación temprana de la VFC ${ }^{23}$. Pero este parece no ser el caso en este estudio, ya que los resultados no muestran reducción del SS hasta Post20. Aparentemente las actividades simpática y parasimpática no siguen las mismas tendencias, provocando que la proporción S:PS presente una respuesta no lineal al efecto del tiempo en la recuperación de la VFC. Se ha reportado que la recuperación post-ejercicio de la VFC alcanza valores de línea de base entre 15 y 30 minutos aproximadamente ${ }^{22}$. Ninguno de los parámetros de VFC analizados en nuestro estudio, Ln RMSSD, Ln SS y el S:PS, alcanzó los niveles de línea de base en Post20. 
Al igual que la reducción de la VFC, se dice que la recuperación de la VFC está afectada principalmente por la intensidad del ejercicio y no por el volumen ${ }^{20,23}$. Varios estudios sostienen que la duración del ejercicio ejecutado por debajo del primer umbral ventilatorio está relacionado con una rápida reactivación postejercicio, pero ésta se retrasa significativamente con intensidades más altas 20,22,23,30. Otros mencionan que la reactivación parasimpática post-ejercicio es una respuesta de entrenamiento individualizada a las especificidades y contenidos del ejercicio ${ }^{5}$. Nuestros resultados concuerdan con la declaración de que la recuperación de la VFC es un proceso multifactorial y que no depende solamente del volumen, la intensidad o incluso la carga interna de entrenamiento ${ }^{31}$. No encontramos relación entre la recuperación de la VFC y ninguna variable de carga de entrenamiento. Los resultados sugieren que para el análisis de la recuperación de la VFC se requiere tomar en cuenta la condición del sujeto, el estado de fatiga y el nivel de entrenamiento ${ }^{22}$

Se dice que el método de TRIMP de Banister no discrimina entre entrenamientos continuos, intermitentes o de intervalo ya que utiliza el promedio de la FCres de la sesión ${ }^{5}$. Hemos ajustados este método adoptando el proceso de Manzi de tomar en cuenta todos y cada uno de los valores de FC observados durante la sesión de entrenamiento y ponderar cada uno de estos valores individualmente con un factor en lugar de promediarlos. Examinamos si ésta modificación presenta una mejor relación con el entrenamiento por intervalos y valores de VFC. Encontramos diferencias mayores en entrenamientos por intervalos en comparación de entrenamientos continuos entre el método de Banister y el método ajustado, aunque estos utilizan el mismo factor de ponderación. Esto sugiere que el método ajustado tiene una mayor sensibilidad para cuantificar cambios de intensidad durante el transcurso del entrenamiento que los valores promedio de FCres. El método de Banister muestra el coeficiente de correlación más bajo con Post5 Ln RMSSD, mientras que los métodos de Edwards y ajustado correlacionan mejor. Parece que cuando el método toma en cuenta los cambios de la intensidad del ejercicio durante el entrenamiento, ya sea en zonas de entrenamiento o considerando cada valor individual de FC, éste ofrece una mejor interpretación del efecto fisiológico del ejercicio sobre el cuerpo.

Se ha explorado el uso de la VFC post-ejercicio como un marcador de carga interna de entrenamiento ${ }^{5,20}$, estableciendo relaciones entre la VFC e índice de esfuerzo percibido (rating of perceived exertion, RPE) como medidas de la intensidad del entrenamiento, sin embargo, no se ha encontrado relación entre el TRIMP de Banister y la VFC, por lo que los resultados previos no recomiendan el uso de la VFC post-ejercicio como un indicador de carga interna de entrenamiento ${ }^{20}$. Nuestros resultados contradicen esta postura, ya que se encontró relación significativa entre Post5 Ln RMSSD y los métodos de TRIMP de Banister, Edwards y ajustado. Esto sugiere que tal vez la reducción de VFC puede ser un indicador de carga interna de entrenamiento. Esta relación se debilita a partir de Post10 hasta Post20, lo que confirma que la recuperación de la VFC no tiene relación con 
parámetros de carga interna de entrenamiento. Los índices de Post5 Ln SS y S:PS no presentaron relación con los métodos de TRIMP. Esto sugiere que la Ln RMSSD es una opción más viable para evaluar la carga interna de entrenamiento que los índices simpático y simpático/parasimpático. Los entrenadores de triatlón pueden considerar la utilización de este método en combinación con valores de VFC en reposo y cálculos de TRIMP para evaluar el estado de fitness-fatiga del triatleta juvenil.

\section{CONCLUSIÓN}

El objetivo de este estudio fue analizar la relación entre la carga interna, cuantificada por varios métodos de TRIMP, con la disminución y recuperación de varios índices de VFC post-ejercicio en triatletas juveniles en mediciones realizadas en campo. La reducción de índices simpáticos, parasimpáticos y de balance simpático/parasimpático de la VFC no solo aporta información objetiva y racional de la intensidad de la sesión de entrenamiento, sino también del volumen y de la carga interna total, evidenciado por la relación que muestra con métodos de TRIMP aceptados como medida de carga interna de entrenamiento. La recuperación de la VFC posterior a un entrenamiento de triatlón es un fenómeno complejo que parece no tener relación con el volumen o la intensidad del ejercicio, sino con el estado de entrenamiento y acondicionamiento del sujeto. Por este motivo, parece inapropiado utilizar este parámetro para evaluar la carga interna de entrenamiento de triatlón juvenil.

\section{REFERENCIAS BIBLIOGRÁFICAS}

1. Meur $Y$ Le, Hausswirth C, Natta F, Couturier A, Bignet F, Vidal PP. A multidisciplinary approach to overreaching detection in endurance trained athletes. $J$ Appl Physiol. 2013;114(3):411-420. doi:10.1152/japplphysiol.01254.2012

2. Banister EW. Modeling Elite Athletic Performance. 2nd ed. (MacDougall JD, Wenger HA, Green HJ, eds.). Champaign, Illinois: Human Kinetics; 1991.

3. Manzi V, lellamo F, Impellizzeri F, Ottavio SD, Castagna C. Relation between Individualized Training Impulses and Performance in Distance Runners. Med Sci Sport Exerc. 2009;41(11):2090-2096. doi:10.1249/MSS.0b013e3181a6a959

4. Akubat I, Abt G. Intermittent exercise alters the heart rate-blood lactate relationship used for calculating the training impulse (TRIMP) in team sport players. J Sci Med Sport. 2011;14(3):249-253. doi:10.1016/j.jsams.2010.12.003

5. Saboul D, Balducci P, Millet GP, Pialoux V, Hautier C. A pilot study on quantification of training load: The use of HRV in training practice. Eur J Sport Sci. 2015;16(2):172-181. doi:10.1080/17461391.2015.1004373

6. Edwards S. The heart rate monitor book. Med Sci Sport Exerc. $1994 ; 26(5): 647$.

7. Lucia A, Hoyos J, Santalla A, Earnest C, Chicharro JL. Tour de France versus 
Rev.int.med.cienc.act.fís.deporte - vol. 20 - número 77 - ISSN: 1577-0354

Vuelta a España: which is harder? Med Sci Sports Exerc. 2003;35(5):872-878. doi:10.1249/01.MSS.0000064999.82036.B4

8. Impellizzeri MF, Rampinini E, Marcora SM. Physiological assessment of aerobic training in soccer. $J$ Sports Sci. 2005;23(6):583-592. doi:10.1080/02640410400021278

9. Alexiou $\mathrm{H}$, Coutts AJ. A comparison of methods used for quantifying internal training load in women soccer players. Int $J$ Sports Physiol Perform. 2008;3(3):320-330. doi:https://doi.org/10.1123/ijspp.3.3.320

10. Stagno K, Thatcher R, Van Someren K. A modified TRIMP to quantify the inseason training load of team sport players. J Sports Sci. 2007;25(6):629-634. doi:10.1080/02640410600811817

11. Manzi V, Castagna C, Padua E, et al. Dose-response relationship of autonomic nervous system responses to individualized training impulse in marathon runners. Am J Physiol Heart Circ Physiol. 2009;296(6):H1733H1740. doi:10.1152/ajpheart.00054.2009.

12. Akubat I, Patel E, Barrett S, Abt G. Methods of monitoring the training and match load and their relationship to changes in fitness in professional youth soccer players. J Sport Sci Med. 2012;30(14):1473-1480. doi:10.1080/02640414.2012.712711

13. Task Force of the European Society of Cardiology the North American Society of Pacing Electrophysiology. Heart Rate Variability Standards of Measurement, Physiological Interpretation, and Clinical Use. Circulation. 1996;93(5):1043-1065. doi:10.1161/01.CIR.93.5.1043

14. Saboul D, Pialoux V, Hautier C. The breathing effect of the LF / HF ratio in the heart rate variability measurements of athletes. Eur J Sport Sci. 2012;14(1):s282-s288. doi:10.1080/17461391.2012.691116

15. Naranjo J, de la Cruz B, Sarabia E, de Hoyo M, Dominguez S. Two New Indexes for the Assessment of Autonomic Balance in Elite Soccer Players. Int $J$ Sport Physiol Perform. 2015;10(4):452-457. doi:https://doi.org/10.1123/ijspp.2014-0235

16. Plews DJ, Laursen PB, Buchheit M. Day-to-day Heart Rate Variability ( HRV ) Recordings in World Champion Rowers: Appreciating Unique Athlete Characteristics. Int $J$ Sport Physiol Perform. 2016;0(0):1-19. doi:10.1123/ijspp.2016-0343

17. Bonet J, Parrado E, Capdevila L. Efectos Agudos del Ejercicio Físico Sobre el Estado de Ánimo y la HRV. Rev Int Med y Ciencias la Act Física y del Deport. 2017;17(65):85-100. doi:http://dx.doi.org/10.15366/rimcafd2017.65.006

18. Plews DJ, Laursen PB, Stanley J, Kilding AE, Buchheit M. Training Adaptation and Heart Rate Variability in Elite Endurance Athletes: Opening the Door to Effective Monitoring. Sport Med. 2013;43(9):773-781. doi:10.1007/s40279013-0071-8

19. Naranjo J, De la Cruz B, Sarabia E, De Hoyo M, Domínguez-Cobo S. Heart Rate Variability: a Follow-up in Elite Soccer Players Throughout the Season. Int J Sports Med. 2015;36(11). doi:10.1055/s-0035-1550047

20. Kaikkonen P, Hynynen E, Mann T, Rusko H, Nummela A. Can HRV be used to evaluate training load in constant load exercises? Eur J Appl Physiol. 
Rev.int.med.cienc.act.fís.deporte - vol. 20 - número 77 - ISSN: 1577-0354

2010;108(3):435-442. doi:10.1007/s00421-009-1240-1

21. Plews DJ, Laursen $P$, Kilding A, Buchheit M. Heart-Rate Variability and Training-Intensity Distribution in Elite Rowers. Int J Sports Physiol Perform. 2014;9(6):1026 - 1032. doi:10.1123/ijspp.2013-0497

22. Seiler S, Haugen $O$, Kuffel E. Autonomic recovery after exercise in trained athletes: intensity and duration effects. Med Sci Sports Exerc. 2007;39(8):1366-1373. doi:10.1249/mss.0b013e318060f17d

23. Kaikkonen P, Hynynen E, Mann T, Rusko H, Nummela A. Heart rate variability is related to training load variables in interval running exercises. Eur J Appl Physiol. 2012;112(3):829-838. doi:10.1007/s00421-011-2031-z

24. Martinmäki K, Rusko H. Time-frequency analysis of heart rate variability during immediate recovery from low and high intensity exercise. Eur J Appl Physiol. 2008;102(3):353-360. doi:10.1007/s00421-007-0594-5

25. Kaikkonen $\mathrm{P}$, Rusko $\mathrm{H}$, Martinmäki K. Post-exercise heart rate variability of endurance athletes after different high-intensity exercise interventions. Scand $J \quad$ Med Sci Sports. 2008;18(4):511-519. doi:10.1111/j.16000838.2007.00728.x

26. Botek M, Mckune AJ, Krejci J, Stejskal P, Gaba A. Change in Performance in Response to Training Load Adjustment Based on Autonomic Activity. Int $J$ Sports Med. 2014;35(06):482-488. doi:10.1055/s-0033-1354385

27. Léger L, Lambert J. A Maximal.Multistage 20-m Shuttle Run Test to Predict VO2 max. Eur J Appl Physiol. 1982;49(1):1-12. doi:https://doi.org/10.1007/BF00428958

28. Goldberger JJ, Le FK, Lahiri M, Kannankeril PJ, Ng J, Kadish AH. Assessment of parasympathetic reactivation after exercise. Am J Physiol Heart Circ Physiol. 2006;290(6):2446-2452. doi:10.1152/ajpheart.01118.2005.

29. Kaikkonen $\mathrm{P}$, Kihu N, Sports $\mathrm{O}$. Heart rate variability dynamics during early recovery after different endurance exercises. Eur $J$ Appl Physiol. 2008;102(1):79-86. doi:10.1007/s00421-007-0559-8

30. Michael S, Jay O, Halaki M, Graham K, Davis G. Submaximal exercise intensity modulates acute post-exercise heart rate variability. Eur $J$ Appl Physiol. 2017;116(4):697-706. doi:10.1007/s00421-016-3327-9

31. Michael S, Graham KS, Oam GMD. Cardiac Autonomic Responses during Exercise and Post-exercise Recovery Using Heart Rate Variability and Systolic Time Intervals - A Review. Front Physiol. 2017;8(1):1-19. doi:10.3389/fphys.2017.00301

Número de citas totales / Total references: 31 (100\%) Número de citas propias de la revista / Journal's own references: 1 (3.2\%)

Rev.int.med.cienc.act.fís.deporte - vol. 20 - número 77 - ISSN: 1577-0354 